\title{
Aktuelle Aspekte zur Epidemiologie, Diagnose und Therapie der Tuberkulose bei HIV-infizierten Patienten
}

\author{
Update on the Epidemiology, Diagnosis and Therapy of Tuberculosis \\ in HIV-infected Patients
}

Autoren

Institute
P. G. Saleeb ${ }^{1,2}$, U. K. Buchwald ${ }^{1,2}$

${ }^{1}$ Division of Infectious Diseases, Department of Medicine, University of Maryland School of Medicine, Baltimore, USA

${ }^{2}$ Institute of Human Virology, University of Maryland School of Medicine, Baltimore, USA eingereicht $\quad 6.5 .2014$ akzeptiert nach Revision 19.6.2014

Bibliografie

Dol http://dx.doi.org/ 10.1055/s-0034-1377514 Pneumologie 2014; 68: 666-675 (c) Georg Thieme Verlag KG Stuttgart · New York ISSN 0934-8387

Korrespondenzadresse Dr. med. Ulrike K. Buchwald Institute of Human Virology, University of Maryland School of Medicine

725 West Lombard Street, S520 Baltimore, MD, 21201

USA

ubuchwald@ihv.umaryland.edu

\section{Zusammenfassung}

$\nabla$

Die Diagnostik und Behandlung der Tuberkulose (TB) bei HIV-infizierten Patienten hat sich in den letzten 10 Jahren weltweit stark verbessert. Dennoch bleibt die Mortalität koinfizierter Patienten erhöht. Der Anteil von HIV-infizierten Patienten an allen TB-Fällen variiert in den europäischen Ländern stark; in Deutschland liegt er bei $4-5 \%$. Die HIV-Infektion beeinflusst die Epidemiologie der TB und die Antibiotikaresistenz. In Ländern mit hoher TB-Inzidenz werden HIV-infizierte Patienten nach Abschluss einer TB-Behandlung oft neu infiziert. Die HIV-Infektion beeinträchtigt viele Immunzellen, die an der TB-Immunabwehr beteiligt sind, und auch eine antiretrovirale Therapie (ART) kann nicht alle Immunfunktionen wiederherstellen. Das klinische Erscheinungsbild der TB bei Patienten mit fortgeschrittener HIV-Infektion ist oft untypisch, und eine disseminierte Erkrankung ist häufig. Neue „Point-of-Care“ Testmethoden versprechen eine Verbesserung der Diagnostik, bedürfen allerdings weiterer Studien. Die Therapie der Koinfektion wird kompliziert durch vielfältige Arzneimittel-Interaktionen und dem Immunrekonstitutionsyndrom (IRIS). Neue Konzepte und Behandlungsstrategien zur Chemoprävention der TB sind insbesondere für HIV-infizierte Patienten erforderlich.

\section{Epidemiologie der TB-|HIV-Koinfektion \\ $\nabla$}

Von 8,6 Millionen neuen Tuberkulose (TB)-Fällen im Jahr 2013 betrafen 1,1 Millionen (13\%) Patienten, die auch an einer HIV-Infektion leiden; $75 \%$ dieser koinfizierten Patienten lebten in Afrika südlich der Sahara [1]. In den letzten zehn Jahren hat sich die Integration von HIV- und TB-Therapie weltweit stark verbessert. Im Jahr 2012 war bei $46 \%$ aller TB-Patienten der HIV-Status be-

\footnotetext{
*Professor Hartmut Lode zum 75. Geburtstag gewidmet.
}

\section{Abstract \\ $\nabla$}

Globally, the diagnosis and treatment of tuberculosis (TB) in HIV-co-infection has improved dramatically over the last 10 years. Nonetheless, the mortality of co-infected patients remains elevated. In European countries, the proportion of HIVinfected patients amongst all TB cases varies greatly; in Germany it is about 4-5\%. HIV-infection changes the molecular epidemiology of TB and the drug resistance situation. In endemic areas, HIV-infected patients are often re-infected after completion of treatment for active TB. HIV has a profound influence on the anti-TB-immune response and antiretroviral therapy (ART) cannot completely restore normal immune function. The clinical presentation in advanced HIV-infection is atypical and disseminated disease is common. New "Point-of-Care" test methods are poised to improve the diagnoses of TB in HIV-infection; however, further research is required. The treatment of co-infection is complicated by drug interactions and the immune reconstitution syndrome (IRIS). New concepts and treatment regimens for chemoprevention of TB are necessary, especially for HIV-infected persons.

kannt; dies war 15-mal mehr als im Jahr 2004 $(\bullet$ Abb. 1); 4,1 Millionen Patienten unter HIVTherapie wurden auf eine aktive TB untersucht, 600000 mehr als im Jahr 2011. 57\% aller koinfizierten Patienten erhielten ein antiretrovirale Therapie (ART), und der Anteil der Patienten, die Isoniazid (INH) zur Therapie einer latenten Tuberkulose-Infektion (LTBI) erhielten, nahm zu $(\bullet$ Abb. 2 und $\odot$ Abb.3). Trotzdem bleibt die Sterblichkeit von koinfizierten Patienten im Vergleich zu TB-monoinfizierten Patienten erhöht ( $\bullet$ Abb.4). 


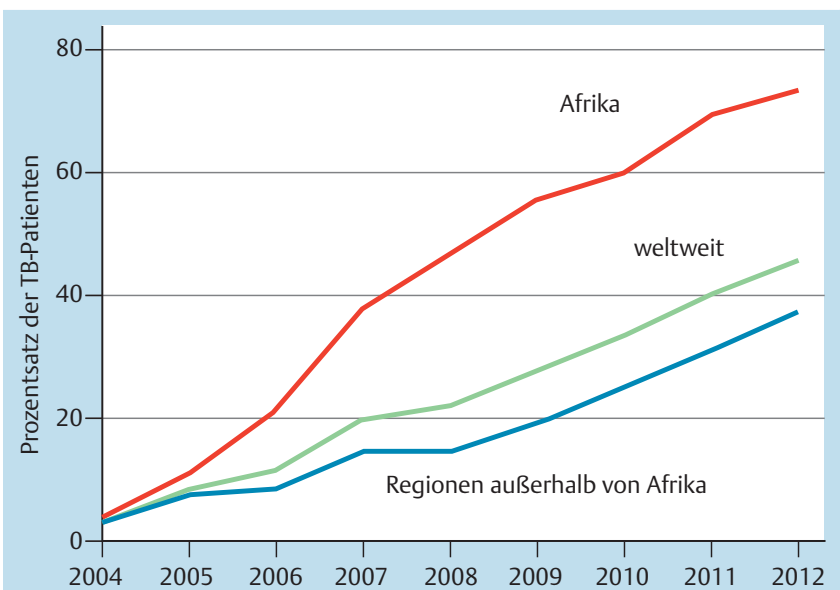

Abb. 1 Prozentsatz der TB-Patienten mit bekanntem HIV-Status, 2004 2012. Reproduziert mit freundlicher Genehmigung der Weltgesundheitsorganisation (WHO) ([1] Abb. 6.2; Seite 69).

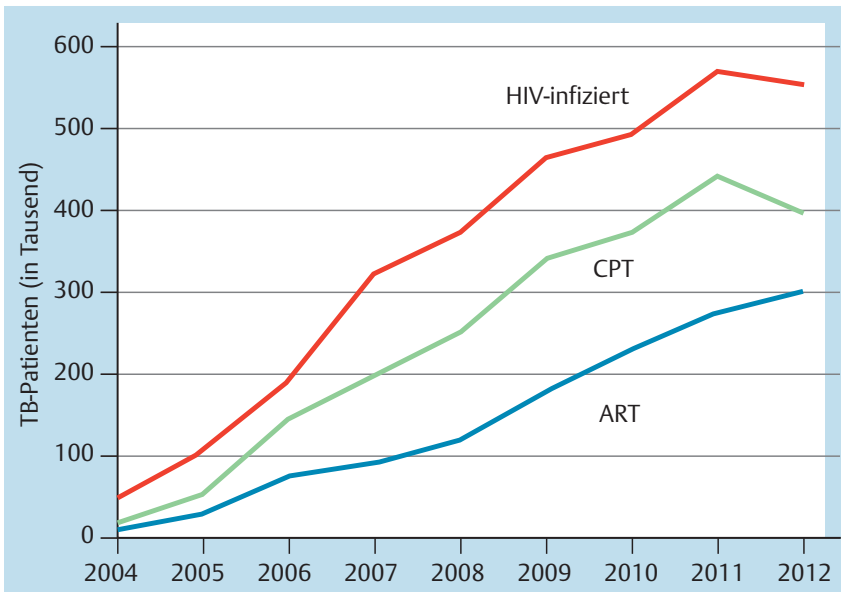

Abb.2 Anzahl der TB-Patienten, die HIV-infiziert sind; Anzahl der TB/HIV koinfizierten Patienten, die eine Cotrimoxazol-Prophylaxe (CPT) oder antiretrovirale Therapie (ART) erhalten, 2004-2012. Reproduziert mit freundlicher Genehmigung der WHO ([1] Abb. 6.4; Seite 71).

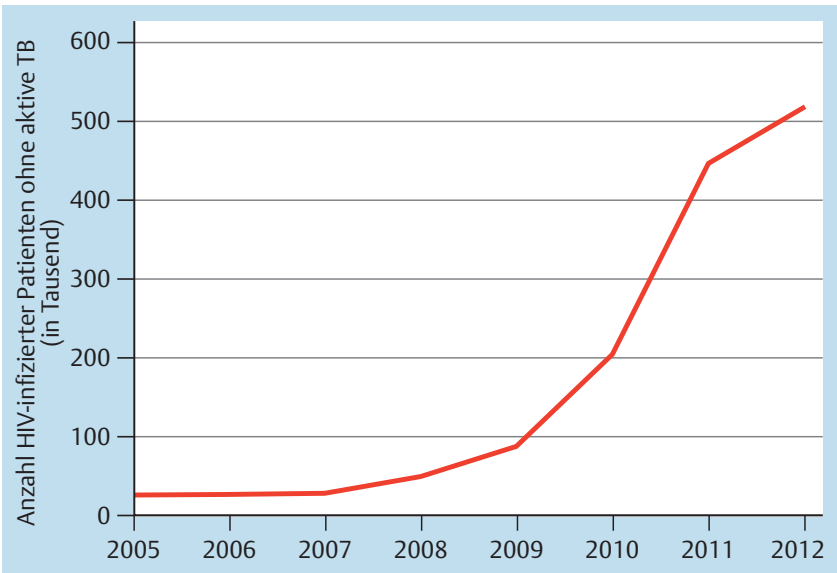

Abb.3 Anzahl der HIV-infizierten Patienten ohne aktive TB, die Isoniazid als Chemoprävention oder Chemoprophylaxe erhalten, 2005-2012. Reproduziert mit freundlicher Genehmigung der WHO ([1] Abb. 6.8; Seite 74).
Die meisten europäischen Länder empfehlen, TB-Patienten routinemäßig auf eine HIV-Infektion zu testen [2]. Nach Schätzungen des European Center for Disease Prevention and Control (ECDC) schwankt jedoch in der Praxis der Prozentsatz der Patienten, die einen HIV-Test erhalten, zwischen 5 und 90\% [3]. Eine uneinheitliche Datenaufzeichnung erschwert genaue Angaben über das Ausmaß der Koepidemie in Europa. Im Jahr 2012 übermittelten nur 16 der 30 Länder des Europäischen Wirtschaftsraumes (EWR) Daten bezüglich der Koinfektion an das ECDC; in diesen 16 Ländern wurden 60,6\% der 35620 TB-Patienten auf eine HIVInfektion untersucht, von denen 5,5\% HIV-positiv waren [3]. In 35 der 53 Länder der Europa-Region der Weltgesundheitsorganisation (WHO), die Daten zur Koinfektion übermitteln, ist der Anteil der TB-Patienten mit HIV-Infektion seit 2004 jährlich angestiegen. Im Jahr 2012 lag der Anteil bei 6,1\% gegenüber 5,4\% im Jahr 2011. In Estland, Litauen, der Ukraine und Portugal waren mehr als 10\% aller TB-Patienten mit HIV infiziert (13,5-16,5\%), in Belgien, den Niederlanden, Spanien, Malta, Russland, der Republik Moldau und Armenien mehr als 5\% (5,2-9,5\%) [3]. Hingegen lag der Anteil in der Slowakei, Slowenien, Island und Bulgarien unter 0,5\%. In Großstädten ist der Anteil der TB-Patienten mit HIV-Infektion oft höher als im landesweiten Durchschnitt. Regionale Unterschiede finden sich auch bezüglich Risikofaktoren wie Drogenmissbrauch und in dem Anteil der im Ausland geborenen Patienten [4].

In Deutschland verhindern Gesetze zum Datenschutz, dass Angaben über eine HIV-Infektion im nationalen Tuberkuloseregister gespeichert werden. Im Jahr 2012 veröffentlichte das Robert Koch-Institut Schätzungen zur HIV-Prävalenz bei deutschen TBPatienten, basierend auf Daten aus verschiedenen Registern [5]. Jährliche Prävalenzraten reichten von 3,5\% im Jahr 2007 bis zu 6,6\% im Jahr 2005 mit einer jüngsten Schätzung von 4\% im Jahr 2009. Koinfizierte Patienten waren im Durchschnitt 38 Jahre alt, und zwei Drittel waren männlich. 59\% der Patienten waren im Ausland geboren, insbesondere in Afrika südlich der Sahara.

In den Vereinigten Staaten ist der Anteil der HIV-infizierten Patienten an allen TB-Fällen von 49\% im Jahr 1993 auf 7\% im Jahr 2012 zurückgegangen. In bestimmten Bevölkerungsgruppen wie zum Beispiel in New York City und unter Zuwanderern sowie bei TB-Patienten im Alter zwischen 25 und 44 Jahren ist der Anteil jedoch höher [6].

\section{Molekulare Epidemiologie der Tuberkulose bei TB-|HIV-Koinfektion \\ $\nabla$}

Die Jahrtausende alte Interaktion von Mycobacterium tuberculosis (MTB) mit dem menschlichen Wirt hat zu adaptiven genetischen Veränderungen in beiden Populationen geführt. Sechs verschiedene MTB-Genotypgruppen weisen eine jeweils charakteristische geografische Verbreitung auf und infizieren bevorzugt Bevölkerungsgruppen dieser Regionen (= sympatrische Übertragung). Diese enge Beziehung überdauert selbst eine Vermischung verschiedener ethnischer Gruppen in kosmopolitischen Städten wie San Francisco und Montreal [7]. In diesem Zusammenhang hat eine aktuelle Studie aus Südafrika eine Assoziation von bestimmten HLA-Genotypen mit Infektionen durch die MTB-Stämme Beijing, LAM, LCC und Quebec gezeigt [8]. Die HIV-Infektion kann jedoch diese stabile Beziehung zwischen dem Wirt und dem Erreger zerstören [9]. So waren Schweizer TB-Patienten mit HIV-Infektion eher mit einem MTB-Stamm infiziert, der nicht ihrer geografischen Herkunft entsprach (allopatrische Übertra- 


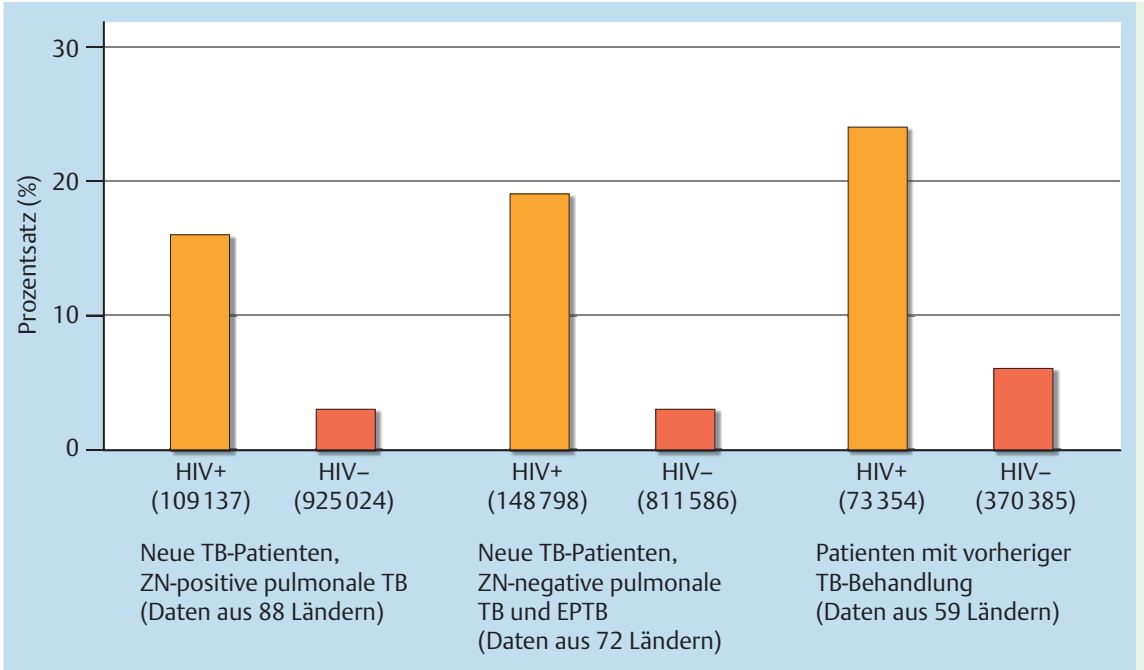

Abb.4 Mortalität in Prozent von HIV-infizierten TB-Patienten im Vergleich zu HIV-negativen TB-Patienten, 2011. Reproduziert mit freundlicher Genehmigung der WHO ([1] Abb. B 3.9.1; Seite 44). $\mathrm{EPTB}=$ extrapulmonale Tuberkulose. gung), unabhängig von ihrem Kontakt zu Ausländern, Reisehäufigkeit, Alter, Geschlecht und Geburtsland.

In Südafrika und Mosambik sind HIV-infizierte Patienten häufiger mit dem Beijing-Stamm infiziert, vermutlich aufgrund der höheren Virulenz und Übertragbarkeit dieses Stammes sowie einer erhöhten Anfälligkeit von HIV-infizierten Personen ihm gegenüber [10]. Eine aktuelle Metaanalyse von 24 Studien in 16 verschiedenen Ländern Europas, Nord- und Südamerikas, Afrikas und Asiens fand eine Assoziation der HIV-Infektion mit einer primär multiresistenten („multi-drug-resistant“, MDR) TB [11]. In Europa ist einer ECDC-Analyse zufolge das Risiko, an einer MDRTB zu erkranken, 2,3-fach höher für HIV-infizierte Patienten als für HIV-negative (95\% Konfidenzinterval 1,68-3,29; p <0,001) [3]. Dies wird überlappenden Risikofaktoren (z.B. Drogenmissbrauch, Obdachlosigkeit) und Übertragungsnetzwerken, aber auch einem schlechteren Therapieansprechen koinfizierter Patienten zugeschrieben.

Der Einfluss der HIV-Infektion auf die Kontagiosität der TB ist umstritten. Eine Metaanalyse von Studien in Kommunen und Krankenhäusern zeigte, dass Patienten mit HIV-Infektion nicht kontagiöser sind als solche ohne die Virusinfektion [12]. In MDR-TB Ausbrüchen hatte jedoch Gesundheitspersonal mit Kontakt zu HIV-infizierten TB-Patienten ein erhöhtes Risiko der Tuberkulin-Haut-Test (THT)-Konversion. In einer Abwandlung des klassischen Meerschweinchen-Experiments zeigten Escombe et al. eine große individuelle Variabilität der Kontagiosität von HIV-positiven TB-Patienten ähnlich wie auch Riley in den ursprünglichen Untersuchungen bei HIV-negativen Patienten [13]. Die reduzierte Rate der Ziehl-Neelsen (ZN)-Positivität und der kavernösen Lungenerkrankungen können einerseits die Kontagiosität von koinfizierten Patienten verringern. Andererseits kann eine atypische Krankheitspräsentation die Diagnose verzögern und ein schlechteres Therapieansprechen die Krankheitsdauer und damit die Zeit, die diese Patienten kontagiös sind, verlängern [14].

In TB-endemischen Gebieten ist ein TB-Rezidiv bei HIV-infizierten Patienten in erster Linie auf eine exogene Reinfektion zurückzuführen, nicht auf eine endogene Reaktivierung [15]. Simultane Infektionen mit mehreren MTB-Stämmen so wie eine gleichzeitige Infektion mit Medikamenten sensiblen und resistenten Stämmen sind beschrieben [16]. Dies kann zur Verbreitung von MDRTB beitragen und unterstreicht die Notwendigkeit für angemessene Infektionsschutzmaßnahmen und präventive Therapiemaß- nahmen bei HIV-infizierten Patienten $[15,17]$. Beide Maßnahmen sind Teil der WHO befürworteten „3 I's“ Initiative („Intensivierte Fallfindung, Infektionsschutzmaßnahmen, Isoniazid Chemoprävention“).

\section{Immunologische Aspekte der TB-|HIV-Koinfektion $\nabla$}

Das Ergebnis einer Infektion mit MTB wird durch das Zusammenspiel von bakterieller Replikation und Immunantwort bestimmt [18]. Heutzutage wird angenommen, dass die TB-Infektion ein Spektrum unterschiedlich stark ausgeprägter Immunkontrolle über die Bakterien umfasst, das von steriler Immunität über Stadien metabolisch inaktiver Bakterien (latente TB), Stadien mit aktiver bakterieller Replikation ohne klinische Symptomatik (subklinische Infektion) bis hin zur klinisch symptomatischen Erkrankung reicht [19]. Viele klinische Symptome der TB werden durch die Immunantwort hervorgerufen. Die HIV-Infektion schwächt diese jedoch ab; daher können sich die Mykobakterien für eine längere Zeit vermehren, ohne klinische Symptome hervorzurufen.

Die HIV-Infektion ist der stärkste Risikofaktor für eine aktive TB, die schon kurz nach einer neuen HIV-Infektion auftreten kann. Eine aktive TB kann bei jeder CD4-Zellzahl auftreten, das Risiko steigt allerdings mit sinkender CD4-Zellzahl an [14]. Selbst bei Patienten mit langjähriger Virussuppression und mit normalen CD4-Zellzahlen unter ART bleibt die TB-Inzidenz um das 2-3fache erhöht im Vergleich zu HIV-negativen Patienten [20]. HIV beeinträchtigt außer den CD4-Zellen noch viele andere Immunzellen, vor allem auch solche des angeborenen Immunsystems wie z.B. die Makrophagen der Atemwege, welche oft die erste Barriere gegenüber inhalierten MTB darstellen ( $\bullet$ Abb.5) [18, $21,22]$. Dazu trägt vor allem die verringerte Apoptose (programmierter Zelltod) von Makrophagen bei, die durch erhöhte IL-10 Konzentrationen mit konsekutiv verminderter TNF- $\alpha$-Produktion sowie durch das HIV-Protein Nef bedingt wird. Dadurch wird die Elimination infizierter Zellen reduziert. Weiterhin vermindert HIV die Azidifizierung des Phagosomes. Die Struktur des Granuloms, des Markenzeichens der fokalen Immunkontrolle über MTB, wird durch HIV grundlegend verändert. Bei koinfizierten Patienten sind granulozytäre Infiltrate und nichtverkäsende Nekrosen häufiger. Das veränderte Zytokin-Milieu im Granulom führt zur Dysfunktion der Immunzellen und begünstigt eine bak- 


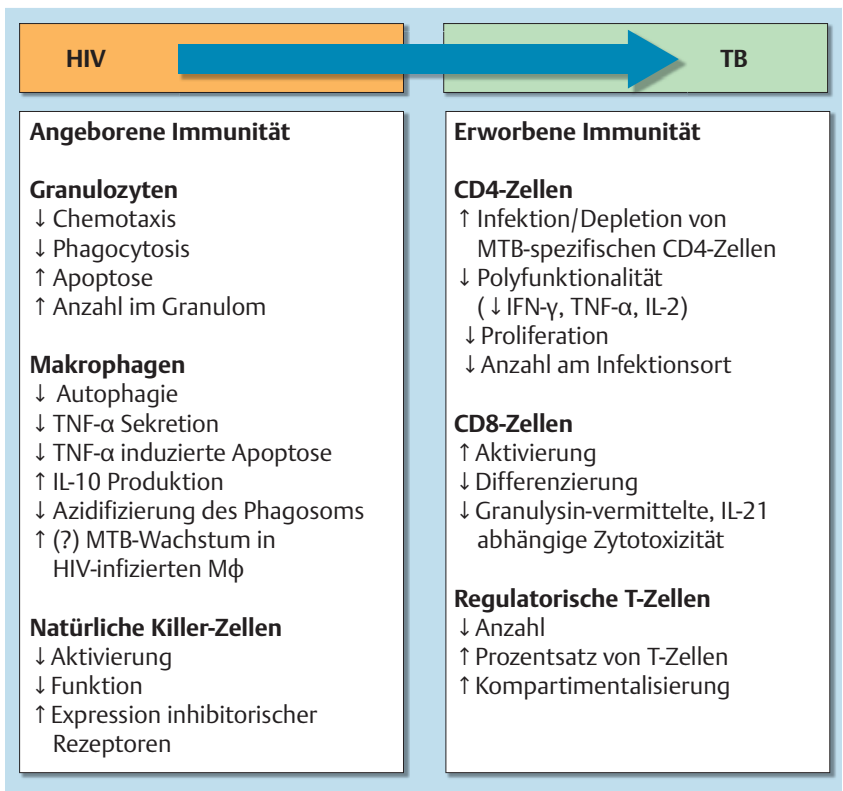

Abb.5 Die HIV-Infektion übt einen wesentlichen Einfluss auf wichtige Immunzellen der angeborenen und erworbenen Immunantwort aus und verringert ihre Abwehrkapazität gegen eine Tuberkulose. MTB = Mycobacterium tuberculosis; $M \varphi=$ Makrophagen [18, 21-23].

\begin{tabular}{|l|}
\hline \multicolumn{1}{|c|}{ TB } \\
\hline $\begin{array}{l}\text { } \text { Expression der HIV-Korezeptoren CCR5 and CXCR4 } \\
\downarrow \text { Sekretion von RANTES als Liganden für CCR5 } \\
\uparrow \text { Aktivierung des HIV-Reservoirs durch TNF- } \alpha \text { und IL-6 } \\
\uparrow \text { HIV-Replikation in MTB-infizierten M } \phi\end{array}$ \\
\hline
\end{tabular}

Abb.6 Die Tuberkulose kann den Verlauf einer HIV-Infektion durch eine gesteigerte Virusreplikation in einem pro-inflammatorischen Zytokinmilieu beschleunigen [18, 21-23].

terielle Replikation. Anstatt die Disseminierung der MTB zu verhindern, tragen die durch die HIV-Infektion modifizierten Granulome zur Verbreitung von MTB im Körper bei.

MTB-spezifische CD4-Zellen sind in den Atemwegen von HIV infizierten Patienten verringert, und jüngste Untersuchungen legen nahe, dass MTB-spezifische CD4-Zellen besonders empfindlich für eine HIV-Infektion sind, da sie eine hohe Anzahl des HIV-Corezeptors CCR5 tragen und als früh differenzierte Zellen besonders viel IL-2 produzieren [23]. Im Vergleich dazu sezernieren CMV-spezifische CD4-Zellen mehr MIP-1 $\beta$, das als Ligand für den CCR5-Rezeptor die Infektion mit HIV kompetitiv verringern kann. Als terminal differenzierte Zellen sind CMV-spezifische CD4-Zellen auch weniger empfindlich für die HIV-Infektion.

Durch die ART werden viele gestörte Immunfunktionen wiederhergestellt, jedoch wird die volle qualitative Funktionalität des Immunsystems oft nicht mehr erreicht. So bleiben trotz Rekonstitution der CD4-Zellzahlen Veränderungen in der phänotypischen Verteilung und Funktion im Vergleich zu HIV-negativen Personen bestehen [18]. Auf der anderen Seite begünstigt eine TB die Progression der HIV-Infektion ( $\bullet$ Abb. 6).

\section{Klinische Präsentation der Tuberkulose bei HIV-infizierten Patienten \\ $\nabla$}

Die Lunge ist auch bei koinfizierten Patienten die häufigste Lokalisation einer TB, jedoch verändert die HIV-Infektion die klinische Präsentation der TB, beschleunigt ihren klinischen Verlauf und erhöht die Sterblichkeit. In endemischen Gebieten ist bei HIV-infizierten Patienten auch ein Husten von $<3$ Wochen Dauer verdächtig auf eine aktive TB. Während Hämoptysen seltener sind, können Fieber und erheblicher Gewichtsverlust häufiger sein und helfen, eine TB von anderen Pneumonien zu unterscheiden [24]. Die diagnostische Sensitivität und Spezifität einer Anamneseerhebung auf klinische Symptome einschließlich Husten, Fieber, Nachtschweiß und Gewichtsverlust variiert in verschiedenen geografischen Gebieten, ist aber Teil der WHO-Strategie zur aktiven Fallsuche (,intensified case finding“) [25].

Patienten mit einer CD4-Zellzahl von $>350-500 / \mathrm{mm}^{3}$ haben ein ähnliches Krankheitsbild wie HIV-negative Personen. Mit sinkenden CD4-Zellzahlen kann die pulmonale TB jedoch atypische Symptome und Befunde aufweisen. In Uganda hatten $21 \%$ der HIV-infizierten TB-Patienten mit einer CD4-Zellzahl von $<50 / \mathrm{mm}^{3}$ eine unauffällige Röntgenthoraxaufnahme gegenüber nur $2 \%$ der Patienten mit CD4-Zellen $>500 / \mathrm{mm}^{3}$ [26]. Ferner hatten $23 \%$ der Patienten mit den niedrigsten CD4-Zellzahlen eine negative ZN-Mikroskopie im Vergleich zu 1\% der Patienten mit CD4-Zellen $>500 / \mathrm{mm}^{3}$. Studien zur aktiven Fallsuche haben Patienten identifiziert, die trotz normaler Röntgenaufnahmen und minimaler klinischer Symptomatik positive Sputumkulturen hatten [27]. Diese subklinische TB-Erkrankung stellt ein großes Problem bei koinfizierten Patienten dar. Post-mortem Studien aus Südafrika legen nahe, dass eine TB in bis zur Hälfte der HIVinfizierten Patienten vorhanden ist, ohne dass sie ante-mortem diagnostiziert wurde [27].

Eine disseminierte TB (aktive TB an mehr als 2 nicht benachbarten Lokalisationen) und eine extrapulmonale TB (EPTB) sind häufig bei koinfizierten Patienten. Eine kombinierte pulmonale und extrapulmonale Erkrankung ist bei $30-70 \%$ der HIV-infizierten Patienten beschrieben, vor allem bei solchen mit niedrigen CD4Zellzahlen, im Vergleich zu nur 5-12\% der HIV-negativen Patienten [27]. Die am häufigsten von EPTB/disseminierter TB betroffenen Organe sind die Milz, Lymphknoten und seröse Häute. Eine Mykobakteriämie ist häufig bei HIV-Infektion und kann mit einem septischen Schock verbunden sein. Bei Patienten mit CD4-Zellzahlen $<100 / \mathrm{mm}^{3}$ sind Blutkulturen in bis zu $49 \%$ der Fällen positiv [27]. Eine Beteiligung der Meningen ist häufiger bei HIV-Koinfektion und kann mit einem Befall anderer Organe assoziiert sein. Die Liquorbefunde sind in der Regel ähnlich wie bei HIV-negativen Personen; azelluläre Befunde sind allerdings beschrieben.

Eine Miliartuberkulose ist auf eine massive lympho-hämatogene Verbreitung von Bakterien zurückzuführen. Bei immunkompetenten Personen tritt sie in etwa 20\% der EPTB und 2-3\% aller TB-Fälle auf. Aufgrund der unspezifischen Symptome ist die Diagnose schwierig. Bei koinfizierten Patienten ist die Erkrankung häufiger, wenn die CD4-Zellen unter $200 / \mathrm{mm}^{3}$ abfallen. Das klinische Bild ist ähnlich wie bei Patienten ohne HIV-Infektion. Bei weit fortgeschrittener HIV-Infektion/AIDS werden jedoch kutane Manifestationen (Papeln oder Vesikel, Tuberkulose Cutis Miliaris Disseminata) und eine mediastinale Lymphadenopathie häufiger gefunden [28]. Tuberkulinanergie, negative Sputum-Abstriche und eine Mykobakteriämie sind ebenfalls häufig. 
Die Sterblichkeit in den ersten Monaten der TB-Behandlung ist bis zu 10-25 mal höher bei TB/HIV-koinfizierten Patienten als bei HIV-uninfizierten Patienten. Dies liegt zum einen in der weiter fortgeschrittenen Erkrankung bei koinfizierten Patienten begründet, zum anderen ist es durch das Vorliegen weiterer opportunistischer Infektionen (OI) bedingt.

\section{Diagnose der aktiven Tuberkulose bei HIV-infizierten Patienten \\ $\nabla$}

In den meisten Endemiegebieten wird die aktive TB mittels ZNMikroskopie diagnostiziert. Bei HIV-infizierten Personen ist der ZN-Abstrich jedoch häufiger negativ und die Sensitivität eines Abstrichs nicht höher als 30\%. Die Fluoreszenzmikroskopie (z. B. mit Auramin-Rhodamin) kann die Sensitivität um etwa $10 \%$ erhöhen [29]. Die ZN-negative Tuberkulose muss initial oft auf Grund des klinischen Verdachts behandelt werden. In Uganda betrug die Sensitivität und Spezifizität des empirischen Therapiebeginns in HIV-infizierten Patienten 63\% und 74\% im Vergleich zur Kultur [30].

Der Goldstandard für die Diagnose der Tuberkulose ist die Kultur auf festen oder in flüssigen Medien, allerdings sind Ergebnisse erst nach 2-6 Wochen erhältlich. Neuere Diagnosemethoden gewinnen daher an Bedeutung, insbesondere bei HIV-infizierten Patienten und in endemischen Gebieten, wo die Qualität der Mikroskopie oft suboptimal ist und die Kultur wegen Mangel an Expertise und Finanzierung nicht zur Verfügung steht. Xpert ${ }^{\circledR}$ MTB/ RIF (Cepheid, USA) ist ein auf einer PCR basierender Test, der innerhalb von 2 Stunden in Sputumproben die Präsenz von MTB und von einer Rifampicin-Resistenz nachweisen kann. Er wird seit 2010 von der WHO als Bestandteil der TB-Diagnose bei HIVinfizierten Patienten oder Verdacht auf MDR-TB empfohlen und ressourcearmen Endemiegebieten kostengünstig zur Verfügung gestellt [31]. Der Test basiert auf der Amplifizierung eines $81 \mathrm{Ba}-$ senpaar langen Segments des RpoB-Gens von MTB. Molekulare Beacons identifizieren Mutationen, die zur Rifampicin-Resistenz führen. Da diese in mehr als $80 \%$ der Fälle in Verbindung mit INH-Resistenz auftreten, kann der Test als Surrogat für die Identifizierung der MDR-TB dienen. Falsch positive Rifampicin-Ergebnisse sind jedoch häufiger in Gebieten mit niedriger MDR-TBPrävalenz. Deshalb muss bei diagnostizierter Rifampicin-Resistenz eine umfassende Resistenzanalyse mittels bakterieller Kultur durchgeführt werden. Der Xpert ${ }^{\circledR}$ MTB/RIF hat eine Spezifität $\geq 98 \%$. Bei HIV-infizierten Patienten mit pulmonaler TB beträgt die Sensitivität eines einzelnen Tests 58-92\% gegenüber $22-73 \%$ für die Mikroskopie [31]. Die Sensitivität ist höher bei Patienten mit längerer Krankheitsdauer und schwerer Symptomatik, wahrscheinlich aufgrund der höheren bakteriellen Last bei diesen Patienten. Der Test verspricht auch eine gute diagnostische Präzision für EPTB, ist allerdings für diese Indikation derzeit nicht zugelassen. Südafrika und Brasilien stellen landesweit die TB-Diagnose von der Mikroskopie auf den Xpert ${ }^{\circledR}$ MTB/RIF Test um, und der globale Roll-Out ist erfreulicherweise sehr hoch [1]. Erste Studien bestätigen seinen Nutzen als POC-Test, der den Therapiebeginn beschleunigt. Ob dies Auswirkungen auf die Morbidität oder Mortalität hat, ist derzeit noch unklar [32]. Ebenso sind Analysen zur Kostengünstigkeit und zur genauen Platzierung des Testes im diagnostischen Algorithmus für TB in verschiedenen ressourcereichen und ressourcearmen Gebieten nicht abgeschlossen [33].
Als POC-Test für die Diagnose der TB bei HIV-infizierten Patienten wurde kürzlich der Alere Determine ${ }^{\mathrm{TM}}$ TB LAM Ag Test (Alere, USA) zugelassen, der das Zellwandlipopolysaccharid Lipoarabinomannan (LAM) innerhalb von 30 Minuten im Urin detektiert. LAM wird bei aktiver TB vor allem bei schwer immunsupprimierten Patienten im Urin ausgeschieden. In einer südafrikanischen Studie von HIV-infizierten Personen mit Kultur-positiver TB betrug die Sensitivität des Tests 67\% für Patienten mit einer CD4Zahl von $<50 / \mathrm{mm}^{3}$, allerdings nur $52 \%$ für Patienten mit CD-Zellen $<100 / \mathrm{mm}^{3}$ [34]. Im Vergleich betrug die Sensitivität der Sputum-Mikroskopie nur 28\%. Eine Kombination von Xpert- und LAM-Tests kann die diagnostische Sensitivität auf 85\% erhöhen, was fast derjenigen der TB-Kultur entspricht (94\%) [35]. Weitere Tests sind in der Entwicklung [1].

\section{Behandlung der Tuberkulose bei HIV-infizierten Patienten \\ $\nabla$}

Angesichts der hohen Morbidität und Mortalität der aktiven TB bei HIV-infizierten Personen ist der sofortige Beginn einer antituberkulösen Therapie zwingend, selbst wenn diagnostische Testergebnisse ausstehen. Alle Patienten sollen auch antiretroviral behandelt werden. Allerdings muss bei gleichzeitiger Behandlung beider Infektionen das höhere Risiko von Medikamententoxizität, Arzneimittel-Interaktionen und IRIS beachtet werden. HIV-infizierte Personen, die bereits ART einnehmen, sollen diese fortsetzen. Eventuell muss die ART allerdings aufgrund von Arzneimittel-Interaktionen umgestellt werden. Der Zeitpunkt der Einleitung der antiretroviralen Therapie bei ART-naiven Patienten mit einer neu behandelten Lungentuberkulose ist gut untersucht. Mehrere große, randomisierte Studien haben gezeigt, dass eine frühzeitige Einleitung der ART die Mortalität deutlich senken kann, insbesondere bei den am stärksten immunsupprimierten Patienten, trotz eines höheren Risikos, dadurch ein Immunrekonstitutionssyndrom (IRIS) auszulösen, und trotz höherer Therapienebenwirkungen ( $\bullet$ Tab.1) [36-39]. Aufgrund dieser Studien empfiehlt die Weltgesundheitsorganisation (WHO) bei HIV-positiven Patienten mit CD4-Zellzahlen $<50 / \mathrm{mm}^{3}$, HAART innerhalb von 2 Wochen nach Beginn der antituberkulösen Behandlung zu beginnen und bei HIV-positiven Patienten mit höheren CD4-Zellzahlen innerhalb von 8 Wochen [25]. Daten zum Beginn der ART bei extrapulmonaler Tuberkulose sind nicht so eindeutig. Ein frühzeitiger Beginn ist möglicherweise von Nachteil für HIV-infizierte Patienten mit einer tuberkulösen Meningitis. Eine Studie in Vietnam randomisierte 253 HIV-infizierte, ART-naive Patienten mit TB-Meningitis zu sofortigem ART-Beginn oder einem um 2 Monate verspäteten Therapiebeginn. Ein frühzeitiger ART Beginn ergab keinen Vorteil bezüglich der Sterblichkeit, aber eine deutlich höhere Rate an schweren Nebenwirkungen [40]. Daher muss die Einleitung der ART bei Patienten mit ZNS- Tuberkulose sehr genau überwacht werden.

Arzneimittel-Wechselwirkungen sind ein großes Problem der Therapie der TB/HIV-Koinfektion, insbesondere wegen der Rifamycine. Rifampicin ist einer der stärksten Induktoren des Cytochrom P450 (insbesondere CYP3A4), wodurch es zu einer Abnahme der Serumspiegel von antiretroviralen Medikamenten kommen kann. Allerdings sind Rifamycine unverzichtbar für die TBBehandlung; werden sie weggelassen, ist das Risiko eines Therapieversagens erhöht [41]. 
Tab. 1 Randomisierte klinische Studien zum optimalen ART-Therapiebeginn bei ART-naiven TB-Patienten [36 - 39].

\begin{tabular}{|c|c|c|c|c|c|c|c|c|}
\hline Studie & Land & $\mathbf{N}$ & $\begin{array}{l}\text { Studienarme: } \\
\text { ART-Beginn }\end{array}$ & $\begin{array}{l}\text { Mortalität } \\
\%\end{array}$ & p-Wert & $\begin{array}{l}\text { IRIS } \\
\%\end{array}$ & p-Wert & Kommentar \\
\hline $\begin{array}{l}\text { SAPiT } \\
{[36,37]}\end{array}$ & Südafrika & 642 & $\begin{array}{l}\text { 1. Früh integriert: } \\
\text { < } 4 \text { Wochen nach } \\
\text { ATT-Beginn } \\
\text { 2. Spät integriert: } \\
\text { <4 Wochen nach Ab- } \\
\text { schluss der intensiven } \\
\text { Phase der ATT } \\
\text { 3. Sequentiell: } \\
4 \text { Wochen nach Ab- } \\
\text { schluss der ATT }\end{array}$ & $\begin{array}{l}\text { Gruppe 1+2: } \\
5,8 \\
12,7\end{array}$ & 0,003 & 8,4 & $<0,001$ & $\begin{array}{l}\text { Mortalität/AIDS-Inzidenzrate in Patienten } \\
\text { mit CD4 < } 50 \text { (pro } 100 \text { Personenjahre) } \\
\text { Gruppe 1: } 8,5 \text {; Gruppe 2: } 26,3 ; p=0,06 \\
\text { Hospitalisierung für IRIS: } \\
\text { Gruppe 1: } 42 \% \text {; Gruppe 2: } 22 \% \text {; Gruppe 3: } \\
5 \% \text {; }=0,01 \\
\text { ART-Wechsel häufiger in Gruppe } 1(4,6 \%) \\
\text { als Gruppe } 2(0,47 \% \text { ) } \\
\text { kein Unterschied in Grad } 3 \text { oder Grad } 4 \text { UAW } \\
\text { (außer IRIS), TB- oder ART-Therapieerfolg }\end{array}$ \\
\hline $\begin{array}{l}\text { CAMELIA } \\
\text { [39] }\end{array}$ & Kambodscha & 661 & $\begin{array}{l}\text { 1. } 2 \text { Wochen nach ATT- } \\
\text { Beginn } \\
\text { 2. } 8-12 \text { Wochen nach } \\
\text { ATT-Beginn }\end{array}$ & $\begin{array}{l}18 \\
27\end{array}$ & 0,002 & $\begin{array}{l}33 \\
14\end{array}$ & $<0,001$ & $\begin{array}{l}6 \text { Todesfälle durch IRIS in Gruppe } 1 \\
\text { kein Unterschied in Grad } 3 \text { oder } 4 \text { UAW } \\
\text { (außer IRIS), TB- oder ART-Therapieerfolg }\end{array}$ \\
\hline $\begin{array}{l}\text { A5221 STRIDE } \\
\text { [38] }\end{array}$ & $\begin{array}{l}\text { 26 Zentren } \\
\text { (4 Kontinente) }\end{array}$ & 809 & $\begin{array}{l}\text { 1. } 2 \text { Wochen nach ATT- } \\
\text { Beginn } \\
\text { 2. } 8-12 \text { Wochen nach } \\
\text { ATT-Beginn }\end{array}$ & $\begin{array}{l}7,7 \\
9,2\end{array}$ & 0,45 & $\begin{array}{l}11 \\
5\end{array}$ & $<0,001$ & $\begin{array}{l}\text { Mortalität oder AIDS definierende Erkran- } \\
\text { kung in Patienten mit CD4 <50: } \\
\text { Gruppe 1: } 15,5 \% \text {; Gruppe 2: } 26,6 \% ; p=0,02 \\
\text { kein Unterschied in Inzidenz von Grad } 3 \text { oder } 4 \\
\text { UAW (außer IRIS), ART-Therapieerfolg oder } \\
\text { TB-Therapiebeendigung }\end{array}$ \\
\hline
\end{tabular}

ATT: Antituberkulöse Therapie

UAW: Unerwünschte Arzneimittelwirkungen

Rifampicin hat erhebliche Wechselwirkungen mit den Non-Nukleosid-Reverse-Transkriptase-Inhibitoren (NNRTIs) Nevirapin und Efavirenz, die weltweit am häufigsten zur Erstlinien-ART eingesetzt werden ( Tab.2) [42]. Pharmakokinetische Studien zeigen, dass Rifampicin die Serumkonzentration von Nevirapin um 20-55\% senkt und die von Efavirenz um 20-30\%. In den meisten klinischen Studien war ein virologisches Therapieversagen häufiger in TB-Patienten, die Rifampicin in Kombination mit Nevirapin erhielten, als solchen, die Efavirenz erhielten. Daher ist Efavirenz der von der WHO empfohlene NNRTI in der Behandlung der Koinfektion [25]. Efavirenz senkt hingegen die Rifabutin-Spiegel durch Induktion von CYP3A4; deshalb muss die Rifabutin-Dosierung erhöht werden; allerdings wird die Kombination mit Rifampicin bevorzugt ( Tab. 2).

Rifampicin senkt auch die Serumkonzentration von Proteaseinhibitoren (PIs). Die Dosis des PI kann daher verdoppelt werden oder der Boost augmentiert werden durch eine höhere Dosis von Ritonavir („Super Boost“); jedoch sind beide Strategien mit Nebenwirkungen, insbesondere einer erhöhten Hepatotoxizität, verbunden [42]. Rifabutin hat einen minimalen Effekt auf Serumkonzentration von PIs und ist daher das Rifamycin der Wahl für HIV-infizierte Patienten, die PIs einnehmen. Allerdings erhöhen PIs die Serumspiegel von Rifabutin, wodurch auch die Toxizität von Rifabutin erhöht wird, z.B. Leukopenie und Uveitis. Es wird empfohlen, die Dosis von Rifabutin von $300 \mathrm{mg}$ pro Tag auf 150 mg pro Tag zu verringern ( $\bullet$ Tab. 2) [42]. Bezüglich der Verwendung von neueren antiretroviralen Medikamenten mit Rifamycinen gibt es einige pharmakokinetische Studien, jedoch wenig klinische Erfahrung. Die NNRTIs Rilpivirin und Etravirin sollten aufgrund verminderter Serumspiegel nicht mit Rifampicin eingesetzt werden. Die Integraseinhibitoren Raltegravir und Dolutegravir können wahrscheinlich in doppelter Dosis mit Rifampicin verabreicht werden ( Tab.2) [42]. Andere Tuberkulostatika haben weniger pharmakokinetische Wechselwirkungen; jedoch sind überlappende Toxizitäten zu beachten ( Tab.3).
Viele internationale Leitlinien empfehlen eine Standardtherapiedauer der TB von 6 Monaten für HIV-infizierte Patienten mit einer unkomplizierten Lungentuberkulose und ggfs. eine Verlängerung auf 9 Monate, wenn die Sputum-Mikroskopie nach 2 Monaten noch positiv ist. Es gibt jedoch Hinweise, dass eine um 2 -3 Monate verlängerte Therapiedauer die Rezidivrate koinfizierter Patienten verringern kann [41]. Hier ist insbesondere die Dauer der Rifamycin Therapie wichtig. In Deutschland wird eine 9-monatige Therapie empfohlen [44]. Die Initialphase von 2 Monaten enthält INH, ein Rifamycin, Ethambutol und Pyrazinamid, die anschließende Kontinuitätsphase INH und ein Rifamycin [44]. Die Therapie der extrapulmonalen TB beträgt je nach Lokalisation 9-12 Monate.

Eine intermittierende Therapie wird international häufig praktiziert, um die Toxizität zu verringern, ist in Deutschland jedoch generell nicht empfohlen [44,45]. Bei HIV-infizierten Patienten sollte sie auf keinen Fall eingesetzt werden, da das Risiko einer erworbenen Rifampicin-Resistenz groß ist [41]. Eine überwachte Behandlung („directly observed therapy“, DOT) ist dringend zu empfehlen.

\section{Das Immunrekonstitutionssyndrom (IRIS) \\ $\nabla$}

Eine Verschlechterung der klinischen Symptome unter antituberkulöser Therapie ist eine bekannte Komplikation auch ohne HIVInfektion, die der Wiederherstellung der zellvermittelten Immunität und ansteigenden TNF- $\alpha$-Spiegel nach MTB-induzierter Immunsuppression zugeschrieben wird [46]. Im Falle einer HIV-Infektion kann der Beginn der ART zu zwei klinischen IRIS-Formen führen: Ein paradoxes („paradoxical“) IRIS tritt auf, wenn sich die Symptome einer anbehandelten opportunistischen Infektion (OI) während der Immunrekonstitution verschlechtern. Es wird angenommen, dass Antigene die Symptome triggern; mikrobiologische Kulturen sind oft negativ. Ein demaskierendes („unmasking“) IRIS ist das Auftreten neuer klinischer Symptome einer zu- 
Tab. 2 Arzneimittel-Interaktionen bei simultaner Therapie der TB und der HIV-Infektion [27, 42].

\begin{tabular}{|c|c|c|c|c|}
\hline Rifamycine & Antiretrovirales Medikament & Interaktion & Empfehlung für ART & Kommentar \\
\hline \multirow[t]{13}{*}{ Rifampicin } & NRTIs & keine & Standarddosis & $\begin{array}{l}\text { reine } 3 \text {-fach Kombination von NRTI nur in } \\
\text { Ausnamefällen empfohlen }\end{array}$ \\
\hline & NNRTIs & & & \\
\hline & Nevirapin (NVP) & NVP $\downarrow 20-55 \%$ & nicht empfohlen & keine Einleitungsphase mit $200 \mathrm{mg}$ täglich \\
\hline & Efavirenz (EFV) & $\mathrm{EFV} \downarrow 20-30 \%$ & EFV $600 \mathrm{mg}$ bis $800 \mathrm{mg} / 24 \mathrm{~h}$ & $\begin{array}{l}\text { einige Experten empfehlen } 800 \mathrm{mg} \text { EFV } \\
\text { für Patienten }>50 \mathrm{~kg}\end{array}$ \\
\hline & Etravirin (ETV) & keine Daten & nicht empfohlen & \\
\hline & Rilpivirin (RPV) & RPV $\downarrow 90 \%$ & nicht empfohlen & \\
\hline & Protease-Inhibitoren (PIs) & $\mathrm{PI} \downarrow 75-90 \%$ & nicht empfohlen & $\begin{array}{l}\text { doppelte Dosis Lopinavir/Ritonavir oder } \\
\text { hochdosierter Ritonavir-Boost zusammen } \\
\text { mit Lopinavir nicht gut verträglich }\end{array}$ \\
\hline & Integrase-Inhibitoren & & & \\
\hline & Raltegravir (RAL) & RAL $\downarrow 60 \%$ & RAL doppelte Dosis $800 \mathrm{mg} / 12 \mathrm{~h}$ & $\begin{array}{l}\text { klinische Erfahrung gering; } \\
\text { enge Therapiekontrolle empfohlen }\end{array}$ \\
\hline & Elvitegravir (EVG)/Cobicistat & EVG $\downarrow$ & nicht empfohlen & \\
\hline & Dolutegravir (ETG) & DVG $\downarrow 54 \%$ & DVG doppelte Dosis $50 \mathrm{mg} / 12 \mathrm{~h}$ & $\begin{array}{l}\text { klinische Erfahrung gering; } \\
\text { enge Therapiekontrolle empfohlen }\end{array}$ \\
\hline & CCR5-Antagonist & & & \\
\hline & Maraviroc (MVC) & MVC $\downarrow>60 \%$ & MVC doppelte Dosis $600 \mathrm{mg} / 12 \mathrm{~h}$ & $\begin{array}{l}\text { klinische Erfahrung gering; im Generellen } \\
\text { nicht empfohlen }\end{array}$ \\
\hline \multirow[t]{13}{*}{ Rifabutin } & NRTI & keine & Standarddosis & \\
\hline & NNRTI & & & \\
\hline & Nevirapin (NVP) & keine & Standarddosis & \\
\hline & Efavirenz (EFV) & Rifabutin $\downarrow 38 \%$ & Standarddosis & $\begin{array}{l}\text { Rifabutin-Dosis erhöht auf } 450-600 \mathrm{mg} / \\
12 \mathrm{~h} \text {; Rifampicin bevorzugt }\end{array}$ \\
\hline & Etravirin (ETV) & ETV $\downarrow 37 \%$ Rifabutin $\downarrow 17 \%$ & Standarddosis & $\begin{array}{l}\text { klinische Erfahrung gering; } \\
\text { enge Therapiekontrolle empfohlen; } \\
\text { nicht in Kombination mit einem PI }\end{array}$ \\
\hline & Rilpivirin (RPV) & RPV $\downarrow 46 \%$ & $\begin{array}{l}\text { RPV in doppelter Dosis von } \\
50 \mathrm{mg} / 24 \mathrm{~h}\end{array}$ & $\begin{array}{l}\text { FDA-Zulassung im Mai } 2014 \text { geändert von } \\
\text { kontraindiziert zu doppelter Dosierung }\end{array}$ \\
\hline & Protease-Inhibitoren (PIs) & Rifabutin $\uparrow \uparrow$ & Standarddosis & $\begin{array}{l}\text { Rifabutin-Dosis erniedrigt auf } 150 \mathrm{mg} / 24 \mathrm{~h} \text {. } \\
150 \mathrm{mg} \text { alle } 2 \text { Tage oder } 3 \times / \text { Woche nicht } \\
\text { mehr empfohlen, da die Rifabutin-Spiegel } \\
\text { eventuell zu niedrig sind }\end{array}$ \\
\hline & Integrase-Inhibitoren & & & \\
\hline & Raltegravir (RAL) & RAL $\uparrow 19 \%$ & Standarddosis & \\
\hline & Elvitegravir (EVG)/Cobicistat & EVG $\downarrow 21 \%$ Rifabutin $\uparrow 600 \%$ & nicht empfohlen & \\
\hline & Dolutegravir (DTG) & keine & Standarddosis & keine klinische Erfahrung \\
\hline & CCR5-Antagonist & & & \\
\hline & Maraviroc (MVC) & MVC eventuell $\downarrow$ & Standarddosis & $\begin{array}{l}\text { keine klinische Erfahrung; einige Experten } \\
\text { empfehlen, die Dosis von MVC auf } \\
600 \mathrm{mg} / 12 \mathrm{~h} \text { zu erhöhen. }\end{array}$ \\
\hline
\end{tabular}

Die Interaktion stellt die Einflussnahme auf die Area Under the Curve (AUC) dar.

NRTI: Nukleos(t)idische Reverse Transkriptase-Inhibitoren; NNRTI: Nicht-Nukleosidische Reverse Transkriptase-Inhibitoren

Tab. 3 Überlappende unerwünschte Arzneimittelwirkungen (UAW) von antiretroviralen Medikamenten und Erstrang-Tuberkulostatika [27,44].

\begin{tabular}{|c|c|c|}
\hline UAW & $\begin{array}{l}\text { Antiretrovirales } \\
\text { Medikament }\end{array}$ & $\begin{array}{l}\text { Erstrang-Tuberkulo- } \\
\text { statika }\end{array}$ \\
\hline Neuropathie & (Stavudin) & Isoniazid, Ethambutol \\
\hline $\begin{array}{l}\text { gastrointestinale } \\
\text { UAW }\end{array}$ & alle & alle \\
\hline Hepatotoxizität & $\begin{array}{l}\text { Efavirenz, Nevirapin, } \\
\text { Pls }\end{array}$ & $\begin{array}{l}\text { Isoniazid, Rifampicin, } \\
\text { Rifabutin, Pyrazinamid }\end{array}$ \\
\hline Myelotoxizität & Zidovudin & $\begin{array}{l}\text { Isoniazid, Rifampicin, } \\
\text { Rifabutin }\end{array}$ \\
\hline kutane UAW & $\begin{array}{l}\text { Abacavir, Nevirapin, } \\
\text { Efavirenz, Darunavir }\end{array}$ & alle \\
\hline Nierenschädigung & Tenofovir & Streptomycin, Rifampicin \\
\hline ZNS-Störungen & Efavirenz & Isoniazid \\
\hline Optikus-Neuritis & (Didanosin) & Ethambutol \\
\hline
\end{tabular}

Die Medikamente in Klammern werden nur noch selten verschrieben. vor nicht diagnostizierten OI aufgrund der Wiederherstellung der erregerspezifischen Immunantworten; Kulturen sind in der Regel positiv. Dieses letztere Syndrom ist von besonderer Bedeutung in Gebieten mit limitierten Ressourcen aufgrund beschränkter diagnostischer Möglichkeiten vor ART-Beginn [27]. Eine niedrige CD4-Zellzahl und eine hohe Viruslast vor Therapiebeginn sind Risikofaktoren. Es wird angenommen, dass IRIS auf eine Dysbalance der Immunantwort gegen mykobakterielle Antigene und einen „Zytokin-Sturm“ zurückzuführen ist. Erhöhte TNF- $\alpha$-, IFN- $\gamma$ - und IL-6-Werte sind beschrieben [18]. Eine gestörte Funktion der natürlichen Killer-Zellen und regulatorischen T-Zellen trägt eventuell auch zur Entstehung von IRIS bei [18].

IRIS ist bei vielen opportunistischen Infektionen beschrieben, neben mykobakteriellen Infektionen vor allem bei der Kryptokokkenmeningitis, dem Kaposi-Sarkom sowie Infektionen mit Herpes-Viren [27]. Inzidenzraten reichen von 8 -45\% [27]. Spezifische Falldefinitionen für ein TB-assoziiertes IRIS sind veröffentlicht [47]. In einer großen südafrikanischen Kohorte von $498 \mathrm{~Pa}-$ 
tienten betraf IRIS 23\% aller Patienten. Die Haut war in $68 \%$ beteiligt, und $44 \%$ waren mit einer schweren OI assoziiert. TB war die häufigste Ätiologie und betraf 34/139 (25\%) der Patienten; $56 \%$ des TB-assoziierten IRIS waren „unmasking“ [48]. Symptome des IRIS sind in der Regel nicht lebensbedrohlich (außer bei ZNSBeteiligung) und mit NSAR oder Steroiden gut behandelbar. Eine randomisierte klinische Studie in Südafrika zeigte, dass Prednison in einer Dosierung von 1,5 mg/ $\mathrm{kg} /$ Tag für 2 Wochen, gefolgt von $0,75 \mathrm{mg} / \mathrm{kg} / \mathrm{Tag}$ für 2 Wochen, Symptome und radiologische Befunde des IRIS verbessern und eine Hospitalisierung verhindern kann [49]. Eine Fortsetzung der ART wird empfohlen. Eine HIV-Diagnosestellung und ein antiretroviraler Therapiebeginn, bevor die CD4-Zellzahl so weit absinkt, dass das Risiko einer OI ansteigt, sowie eine gründliche Diagnostik auf mögliche OIs vor ART-Beginn kann helfen, IRIS zu vermeiden [27].

\section{Diagnose und Therapie der latenten Tuberkulose (LTBI) bei HIV-infizierten Patienten \\ $\nabla$}

Immunkompetente Personen mit LTBI haben ein 5 bis 10\% Risiko, in ihrem Leben an einer aktiven TB zu erkranken; das Risiko ist am höchsten in den ersten 2 Jahren nach der TB-Infektion. Für HIV-infizierte Patienten mit LTBI beträgt das Risiko einer aktiven TB jedoch 5-30\% jährlich und eine primär progressive Tuberkulose ist häufiger [50].

Die Diagnose der LTBI ist schwierig bei koinfizierten Patienten, da die Sensitivität des Tuberkulin-Haut-Tests (THT) und der IGRAs durch die Immunsuppression reduziert ist. Für den THT gilt das auch, wenn der Cut-off für Positivität auf $5 \mathrm{~mm}$ verringert wird, da Anergie häufig ist [51]. Wie auch bei HIV-negativen Patienten ist die Kreuzreaktivität mit dem Bacille-Calmette-Guérin (BCG)Impfstoff und nichttuberkulösen Mykobakteriosen ein Problem. Interferon-Gamma-Release-Assays (IGRAs) messen die IFN- $\gamma$ Freisetzung von peripheren Lymphozyten, die mit den MTB-spezifischen Antigenen der RD1-Region stimuliert werden. Metaanalysen haben gezeigt, dass die Spezifität für die Diagnose einer LTBI für alle IGRAs $>90 \%$ beträgt, auch bei BCG-geimpften Patienten [52]. Allerdings scheint die Sensitivität bei HIV-infizierten Patienten nicht höher zu sein als die des THT, besonders in endemischen Gebieten [52]. Mit sinkender CD4-Zellzahl ist eine ungültige Positivkontrolle häufiger. Wie auch der THT können IGRAs die aktive TB nicht von einer LTBI unterscheiden oder voraussagen, welche Patienten mit LTBI das höchste Risiko haben, an einer aktiven TB zu erkranken, und daher am meisten von der LTBITherapie profitieren würden. Nationale und supranationale Leitlinien für HIV-positive wie HIV-negative Patienten variieren in den Empfehlungen, in welcher Kombination THT und IGRAs anzuwenden sind [53]. In Deutschland kann der THT und/oder ein IGRA angewendet werden [44]. Alle HIV-infizierten Patienten sollen zumindest bei der Erstuntersuchung auf eine LTBI untersucht werden.

Wenn der THT oder IGRA positiv ist, muss eine aktive TB ausgeschlossen werden. Besonders bei Patienten mit niedriger CD4Zellzahl muss dafür eventuell auch bei normaler Röntgenthoraxaufnahme eine Sputumuntersuchung angefordert werden, insbesondere, wenn klinische Symptome wie Husten, Fieber oder Nachtschweiß vorhanden sind [54]. Als Therapieregime für die Chemoprävention der LTBI bei HIV-positiven Personen werden INH für 9 Monate sowie Rifampicin für 4 Monate empfohlen [45]. Einmal wöchentlich verabreichtes INH plus Rifapentin für 12 Wochen zeigte eine ähnliche Wirksamkeit wie INH alleine in Be-

\section{Fazit für die Praxis}

- Auch in Europa ist die HIV-Infektion ein wichtiger Risikofaktor für eine TB-Erkrankung. Jeder TB-Patient muss auf eine HIV-Infektion untersucht werden. HIV-infizierte Personen müssen auf eine LTBI untersucht werden.

- Eine pulmonale und extrapulmonale TB kann sich bei HIVinfizierten Patienten atypisch präsentieren. Bei der Anamnese ist besonders auf Symptome wie Husten, Gewichtsverlust und Nachtschweiß zu achten. Wenn die CD4-Zellzahlen auf $<300 / \mathrm{mm}^{3}$ abfallen, sind Kavernen und ein Befall der Lungenoberlappen seltener. Bei CD4-Zellzahlen von $<100 / \mathrm{mm}^{3}$ finden sich oft Pleuraergüsse und miliare Krankheitsherde.

- Die Sputum-Mikroskopie ist weniger sensitiv bei HIV-infizierten Patienten. Eine Kultur muss angefordert werden. Neuere diagnostische Methoden wie der Xpert ${ }^{\circledR}$ MTB/Rif können zur Unterstützung herangezogen werden.

- Der klinische Verlauf der TB bei HIV-Infektion ist aggressiver. Die antituberkulöse Therapie muss daher unverzüglich, eventuell auch auf reinen klinischen Verdacht hin, begonnen werden. Eine ART sollte in den ersten 2 - 8 Wochen begonnen werden unter Konsultation eines HIV-Mediziners.

zug auf die Reduzierung des Risikos für TB-Reaktivierung bei HIV-negativen und HIV-positiven Patienten $[55,56]$. Der Vorteil dieser Therapie ist jedoch die kürzere Behandlungsdauer mit einer potenziell besseren Therapieadhärenz. Daten über die Interaktion zwischen Rifapentin und der ART sind jedoch unzureichend. Es wird daher empfohlen, dieses Regime nicht bei HIV-positiven Personen, die ART erhalten, anzuwenden, bis weitere Informationen verfügbar sind [56]. In Ländern mit hoher TB/HIVPrävalenz wird INH unabhängig von dem THT-Ergebnis häufig für alle HIV-infizierten Patienten verschrieben, sobald eine aktive TB ausgeschlossen ist, was gesundheitspolitisch eine kombinierte prophylaktische und präventive Maßnahme darstellt [25]. Klinische Studien haben gezeigt, dass eine Verlängerung der INH-Therapie (z.B. auf 36 Monate) die Wirksamkeit erhöht, allerdings erlischt die protektive Wirkung oft, sobald die Gabe von INH abgebrochen wird [57]. In einer Modellanalyse ist dargestellt worden, dass INH bei koinfizierten Personen die LTBI nicht ausheilen kann und auch aufgrund des Risikos einer Reinfektion eine kontinuierliche Gabe nötig ist [58]. Die subklinische TB-Erkrankung und die Häufigkeit einer Reinfektion als Ursache für ein TB-Rezidiv fordern dazu auf, die Konzepte der LTBI-Behandlung zu überdenken [50]. Die ART ist bei HIV-infizierten Patienten auch als präventive Strategie anzusehen, da sie durch die Immunrekonstitution das Risiko einer LTBI-Reaktivierung reduzieren kann.

\section{Danksagung}

Herrn Dr. Dirk Buchwald und Herrn Dr. Rolf-Michael Walther wird herzlich gedankt für ihre Unterstützung bei der Korrekturlesung.

\section{Interessenkonflikt \\ $\nabla$}

Die Autoren geben an, dass kein Interessenkonflikt besteht. 


\section{Literatur}

1 World Health Organization (WHO): Global Tuberculosis Report 2013. http://www.who.int/tb/publications/global_report/en/

2 Kruijshaar ME, Pimpin L, Abubakar I et al. The burden of TB-HIV in the EU: how much do we know? A survey of surveillance practices and results. Eur Respir J 2011; 38: 1374 - 1381

3 World Health Organization (WHO): Tuberculosis surveillance and monitoring in Europe. 2014: http://www.ecdc.europa.eu/en/publications/Publications/tuberculosis-surveillance-monitoring-Europe-2014. pdf

4 Pimpin L, Drumright LN, Kruijshaar ME et al. Tuberculosis and HIV coinfection in European Union and European Economic Area countries. Eur Respir J 2011; 38: 1382 - 1392

5 Fiebig L, Kollan C, Hauer B et al. HIV-prevalence in tuberculosis patients in Germany, 2002-2009: an estimation based on HIV and tuberculosis surveillance data. PLoS One 2012; 7: e49111

6 Trieu L, Li J, Hanna DB et al. Tuberculosis rates among HIV-infected persons in New York City, 2001-2005. Am J Public Health 2010; 100: $1031-1034$

7 Gagneux S, DeRiemer K, Van T et al. Variable host-pathogen compatibility in Mycobacterium tuberculosis. Proc Natl Acad Sci U S A 2006; 103: $2869-2873$

8 Salie $M$, van der Merwe L, Möller $M$ et al. Associations between human leukocyte antigen class I variants and the Mycobacterium tuberculosis subtypes causing disease. J Infect Dis 2014; 209: 216-223

9 Fenner L, Egger M, Bodmer T et al. HIV infection disrupts the sympatric host-pathogen relationship in human tuberculosis. PLoS Genet 2013; 9: e1003318

10 Viegas SO, Machado A, Groenheit $R$ et al. Mycobacterium tuberculosis Beijing genotype is associated with HIV infection in Mozambique. PLoS One 2013; 8: e71999

11 Suchindran S, Brouwer ES, Van Rie A. Is HIV infection a risk factor for multi-drug resistant tuberculosis? A systematic review. PLoS One 2009; 4: e5561

12 Cruciani $M$, Malena $M$, Bosco $O$ et al. The impact of human immunodeficiency virus type 1 on infectiousness of tuberculosis: a meta-analysis. Clin Infect Dis 2001; 33: 1922 - 1930

13 Escombe AR, Moore DA, Gilman RH et al. The infectiousness of tuberculosis patients coinfected with HIV. PLoS Med 2008; 5: e188

14 Kwan CK, Ernst JD. HIV and tuberculosis: a deadly human syndemic. Clin Microbiol Rev 2011; 24: 351 - 376

15 Narayanan S, Swaminathan S, Supply P et al. Impact of HIV infection on the recurrence of tuberculosis in South India. J Infect Dis 2010; 201: $691-703$

16 Warren RM, Victor TC, Streicher EM et al. Patients with active tuberculosis often have different strains in the same sputum specimen. Am J Respir Crit Care Med 2004; 169: 610-614

17 Andrews JR, Gandhi NR, Moodley $P$ et al. Exogenous reinfection as a cause of multidrug-resistant and extensively drug-resistant tuberculosis in rural South Africa. J Infect Dis 2008; 198: 1582-1589

18 Walker NF, Meintjes G, Wilkinson RJ. HIV-1 and the immune response to TB. Future Virol 2013; 8: 57-80

19 Meintjes G, Wilkinson RJ. Undiagnosed active tuberculosis in HIV-infected patients commencing antiretroviral therapy. Clin Infect Dis 2010; 51: 830-832

20 Gupta A, Wood R, Kaplan $R$ et al. Tuberculosis incidence rates during 8 years of follow-up of an antiretroviral treatment cohort in South Africa: comparison with rates in the community. PLoS One 2012; 7: e34156

21 Diedrich CR, Flynn JL. HIV-1/mycobacterium tuberculosis coinfection immunology: how does HIV-1 exacerbate tuberculosis? Infect Immun 2011; 79: 1407-1417

22 Pawlowski A, Jansson M, Sköld $M$ et al. Tuberculosis and HIV co-infection. PLoS Pathog 2012; 8: e1002464

23 Geldmacher C, Ngwenyama N, Schuetz A et al. Preferential infection and depletion of Mycobacterium tuberculosis-specific CD4 T cells after HIV-1 infection. J Exp Med 2010; 207: 2869-2881

24 Selwyn PA, Pumerantz AS, Durante A et al. Clinical predictors of Pneumocystis carinii pneumonia, bacterial pneumonia and tuberculosis in HIV-infected patients. AIDS 1998; 12: 885-893

25 World Health Organization (WHO). WHO policy on collaborative TB/ HIV activities: guidelines for national programs and stakeholders. 2012: $\quad$ http://www.who.int/tb/publications/2012/tb_hiv_policy_9789241503006/en/
26 Chamie G, Luetkemeyer A, Walusimbi-Nanteza M et al. Significant variation in presentation of pulmonary tuberculosis across a high resolution of CD4 strata. Int J Tuberc Lung Dis 2010; 14: 1295-1302

27 Schutz C, Meintjes G, Almajid F et al. Clinical management of tuberculosis and HIV-1 co-infection. Eur Respir J 2010; 36: 1460 - 1481

28 Ray S, Talukdar A, Kundu S et al. Diagnosis and management of miliary tuberculosis: current state and future perspectives. Ther Clin Risk Manag 2013; 9: 9-26

29 Reid MJ, Shah NS. Approaches to tuberculosis screening and diagnosis in people with HIV in resource-limited settings. Lancet Infect Dis 2009; 9: $173-184$

30 Nakiyingi L, Bwanika JM, Kirenga B et al. Clinical predictors and accuracy of empiric tuberculosis treatment among sputum smear-negative HIV-infected adult TB suspects in Uganda. PLoS One 2013; 8: e74023

31 Lawn SD, Mwaba P, Bates $M$ et al. Advances in tuberculosis diagnostics: the Xpert MTB/RIF assay and future prospects for a point-of-care test. Lancet Infect Dis 2013; 13: 349-361

32 Theron G, Zijenah L, Chanda D et al. Feasibility, accuracy, and clinical effect of point-of-care Xpert MTB/RIF testing for tuberculosis in primary-care settings in Africa: a multicentre, randomised, controlled trial. Lancet 2014; 383: 424-435

33 Sohn $H$, Aero AD, Menzies $D$ et al. Xpert MTB/RIF testing in a low tuberculosis incidence, high-resource setting: limitations in accuracy and clinical impact. Clin Infect Dis 2014; 58: 970-976

34 Lawn SD, Kerkhoff AD, Vogt M et al. Diagnostic accuracy of a low-cost, urine antigen, point-of-care screening assay for HIV-associated pulmonary tuberculosis before antiretroviral therapy: a descriptive study. Lancet Infect Dis 2012; 12: 201 - 209

35 Shah $M$, Ssengooba W, Armstrong $D$ et al. Comparative performance of urinary lipoarabinomannan assays and Xpert MTB/RIF in HIV-infected individuals with suspected tuberculosis in Uganda. AIDS 2014

36 Abdool Karim SS, Naidoo K, Grobler A et al. Timing of initiation of antiretroviral drugs during tuberculosis therapy. N Engl J Med 2010; 362: $697-706$

37 Abdool Karim SS, Kogieleum N, Grobler A et al. Integration of antiretroviral therapy with tuberculosis treatment. N Engl J Med 2011; 365: $1492-1501$

38 Havlir DV, Kendall MA, Ive $P$ et al. Timing of antiretroviral therapy for HIV-1 infection and tuberculosis. N Engl J Med 2011; 365: 1482 - 1491

39 Blanc FX, Sok T, Laureillard D et al. Earlier versus later start of antiretroviral therapy in HIV-infected adults with tuberculosis. N Engl J Med 2011; 365: $1471-1481$

40 Török ME, Yen NT, Chau TT et al. Timing of initiation of antiretroviral therapy in human immunodeficiency virus (HIV)-associated tuberculous meningitis. Clin Infect Dis 2011; 52: 1374-1383

41 Khan FA, Minion J, Pai M et al. Treatment of active tuberculosis in HIVcoinfected patients: a systematic review and meta-analysis. Clin Infect Dis 2010; 50: 1288 - 1299

42 Center for Disease Control and Prevention (CDC). Managing Drug Interactions in the treatment of HIV-related tuberculosis. 2013: http://www.cdc.gov/tb/publications/guidelines/tb_hiv_drugs/default. htm

43 Manosuthi $W$, Sungkanuparph S, Tantanathip $P$ et al. A randomized trial comparing plasma drug concentrations and efficacies between 2 nonnucleoside reverse-transcriptase inhibitor-based regimens in HIVinfected patients receiving rifampicin: the N2R Study. Clin Infect Dis 2009; 48: 1752 - 1759

44 Schaberg T, Bauer T, Castell S et al. [Recommendations for therapy, chemoprevention and chemoprophylaxis of tuberculosis in adults and children. German Central Committee against Tuberculosis (DZK), German Respiratory Society (DGP)]. Pneumologie 2012; 66: 133-171

45 Department of Health and Human Services Panel on Antiretoviral Guidelines for Adults and Adolescents. Guidelines for the prevention and treatment of opportunistic infections in HIV-infected adults and adolescents. 2014: http://aidsinfo.nih.gov/guidelines/html/4/adultand-adolescent-oi-prevention-and-treatment-guidelines $/ 0$

46 Choremis CB, Padiatellis C, Zou Mbou Lakis D et al. Transitory exacerbation of fever and roentgenographic findings during treatment of tuberculosis in children. Am Rev Tuberc 1955; 72: 527-536

47 Meintjes G, Lawn SD, Scano F et al. Tuberculosis-associated immune reconstitution inflammatory syndrome: case definitions for use in resource-limited settings. Lancet Infect Dis 2008; 8: 516-523

48 Haddow LJ, Moosa MY, Mosam A et al. Incidence, clinical spectrum, risk factors and impact of HIV-associated immune reconstitution inflammatory syndrome in South Africa. PLoS One 2012; 7: e40623 
49 Meintjes G, Wilkinson RJ, Morroni $C$ et al. Randomized placebo-controlled trial of prednisone for paradoxical tuberculosis-associated immune reconstitution inflammatory syndrome. AIDS 2010; $24: 2381$ 2390

50 Zumla A, Atun R, Maeurer M et al. Viewpoint: Scientific dogmas, paradoxes and mysteries of latent Mycobacterium tuberculosis infection. Trop Med Int Health 2011; 16: 79-83

51 Markowitz N, Hansen NI, Wilcosky TC et al. Tuberculin and anergy testing in HIV-seropositive and HIV-seronegative persons. Pulmonary Complications of HIV Infection Study Group. Ann Intern Med 1993; 119: $185-193$

52 Cattamanchi $A$, Smith $R$, Steingart $K R$ et al. Interferon-gamma release assays for the diagnosis of latent tuberculosis infection in HIV-infected individuals: a systematic review and meta-analysis. J Acquir Immune Defic Syndr 2011; 56: 230 - 238

53 Denkinger CM, Dheda K, Pai $M$. Guidelines on interferon- $\gamma$ release assays for tuberculosis infection: concordance, discordance or confusion? Clin Microbiol Infect 2011; 17: 806-814
54 Cain KP, McCarthy KD, Heilig CM et al. An algorithm for tuberculosis screening and diagnosis in people with HIV. N Engl J Med 2010; 362: $707-716$

55 Martinson NA, Barnes GL, Moulton LH et al. New regimens to prevent tuberculosis in adults with HIV infection. N Engl J Med 2011; 365: $11-20$

56 Recommendations for use of an isoniazid-rifapentine regimen with direct observation to treat latent Mycobacterium tuberculosis infection. MMWR 2011; 60: 1650 - 1653

57 Samandari T, Agizew TB, Nyirenda $S$ et al. 6-month versus 36-month isoniazid preventive treatment for tuberculosis in adults with HIV infection in Botswana: a randomised, double-blind, placebo-controlled trial. Lancet 2011; 377: 1588 - 1598

58 Houben RM, Sumner T, Grant AD et al. Ability of preventive therapy to cure latent Mycobacterium tuberculosis infection in HIV-infected individuals in high-burden settings. Proc Natl Acad Sci U S A 2014; 111: $5325-5330$ 